\title{
The $\eta^{\prime}$ mass on $2+1$ flavor DWF lattices
}

\author{
Duo Guo*t \\ Department of Physics, Columbia University, New York, NY 10027, USA \\ E-mail: dg2806ecolumbia.edu
}

The large $\eta^{\prime}$ mass depends on the topological properties of the QCD gauge fields and an accurate measurement requires an ensemble that well samples the possible topologies of the gauge fields. In this proceedings, we present our analysis of the RBC-UKQCD $24^{3} \times 64 \times 24$ physical coarse DWF ensemble. The evolution of the global topological charge is measured using the 5-loop improved method [1] after cooling via Wilson flow. The results for closed quark loops, which are sensitive to the topology on the original (unflowed) configurations, are shown to have long autocorrelations. Several eigenvectors with small eigenvalues are calculated for one specific configuration and topological fluctuations are found. Preliminary calculation of the $\eta$ and $\eta^{\prime}$ mass is done. Our results show that good sampling of the global topological charge (measured after the Wilson flow) does not necessarily mean rapid evolution of local topological fluctuations.

The 36th Annual International Symposium on Lattice Field Theory - LATTICE2018

22-28 July, 2018

Michigan State University, East Lansing, Michigan, USA.

\footnotetext{
${ }^{*}$ Speaker.

$\dagger$ The author is grateful to Robert M. Mawhinney, David J. Murphy and Chulwoo Jung for helpful discussion in support of this work. Calculations were performed using the Blue Gene/Q computers of the RIKEN-BNL Research Center and Brookhaven National Lab. The software used includes the CPS QCD code, supported in part by the USDOE SciDAC program, the BAGEL assembler kernel generator for high-performance optimized kernels and fermion solvers. The author is supported in part by U.S. DOE grant \#DE-SC0011941.
} 


\section{Introduction}

The large difference between the mass of $\eta$ particle and $\eta^{\prime}$ particle is explained by the fact that the $\mathrm{U}_{\mathrm{A}}(1)$ chiral symmetry of massless $\mathrm{QCD}$ is broken in the QCD vacuum. The reason that the $U_{A}(1)$ symmetry is broken is that the $Q C D$ vacuum has non-trivial topological properties [2]. Specifically, QCD gauge field configurations can have non-zero topological charge. For such configurations, the topological properties are manifested in fermionic fields by the zero-modes of the Dirac operator. These zero-modes of the Dirac operator effect the closed quark loops that enter the correlator for the $\eta^{\prime}$.

Therefore, as part of the measurement of the the mass of the $\eta$ and $\eta^{\prime}$ and the analysis of the reliability of the calculation, we examine the details of the topological properties of our ensemble. In Section 2, we introduce our ensemble and show the topological charge of the configurations in it. In Section 3, we show our results for closed quark loops and discuss their autocorrelations. In Section 4, we present eigenvectors with small eigenvalues for one gauge configuration. (This result was not available at the time of the conference.) Preliminary results for the $\eta$ and $\eta^{\prime}$ mass are shown in Section 5 and conclusions and outlook are given in Section 6.

\section{Lattices and topology evolution}

We work with lattices from the physical coarse ensemble generated by the RBC-UCQCD collaboration [4]. The action for the lattices is the Iwasaki+DSDR (Dislocation Suppressing Determinant Ratio) gauge action. The DSDR part of our action is designed to suppress changes in topology, which is vital to use domain wall fermions at strong coupling. For fermions, we use Mobius domain wall fermions, with two light quarks and one heavy quark. The coupling constant $\beta$ is 1.633 . The input heavy quark mass is 0.085 and light quark mass is 0.00107 . The pion mass measured for this ensemble is around $140 \mathrm{MeV}$ and the kaon mass is around $500 \mathrm{MeV}$ which means that this is a physical ensemble. The lattice size is $24^{3} \times 64 \times 24$, with $a^{-1}=0.98 \mathrm{GeV}$ and periodic boundary conditions on the gauge field. The large lattice spacing promotes changes in topological charge and the DSDR term suppresses them and the balance between them is vital for good sampling of topology and measuring the $\eta^{\prime}$ mass.

Our measurements start from 300th trajectory when the lattice is thermalized. We then measure on every 4th trajectory until trajectory 2236. In total, we measured on 484 configurations.

The global topological charge as a function of trajectory number or molecular dynamics time (MD time) is shown in Figure 1. The gauge field is smoothed by running a Wilson flow. We took $d t=0.05$ and ran for 1000 steps yielding a stable topological charge. The topological charge is measured by the $5 \mathrm{Li}$ method [1]. In Figure 1, we show the topological charge for each trajectory and the running average topological charge. As one can see, the global topological charge is changing by a large amount and the average value is close to zero. From this perspective, our gauge fields are well sampled over all topological sectors and there is no topological freezing. However, as we look at the results for closed quark loops and eigenvectors, we will find a more complicated situation. 


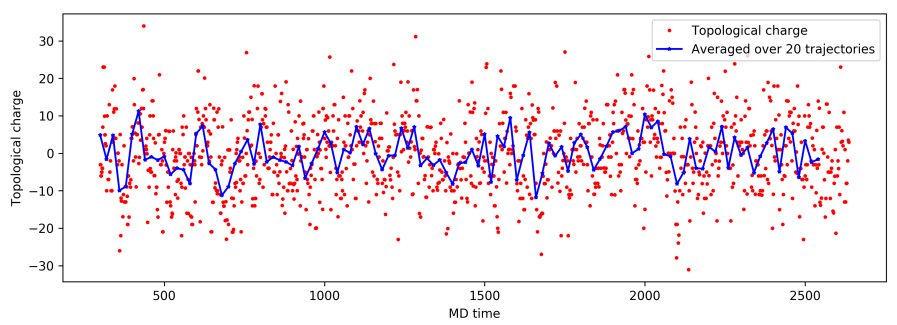

Figure 1: Topological evolution of the lattices

\section{Closed quark loops}

In the calculation of the $\eta^{\prime}$ mass, we must consider $\left\langle\bar{\psi} \gamma_{5} \psi(x) \bar{\psi} \gamma_{5} \psi(y)\right\rangle$, which leads to disconnected terms like $D_{l l}=\left\langle\bar{\psi} \gamma_{5} \psi(x) \bar{\psi} \gamma_{5} \psi(y)\right\rangle$. This leads us to consider closed quark loops on each time slice. During the measurement, we first apply Coulomb gauge to the fields. We then calculate $\sum_{\vec{x}}\left\langle\bar{\psi}(\vec{x}, t) \gamma_{5} \psi(\vec{x}, t)\right\rangle$ using a wall sink and a wall source. The results for the light quark mass are shown in Figure 2 as a function of time slice. Error bars are shown with unbinned data and a binning of 10 (40 MD time units). Note that since we have periodic boundary conditions, the results on each time slice should be similar. However, there is no translational invariance in time direction for our closed quark loop results. What's more, since the average topological charge should be zero, the values of closed quark loops should be zero. However, it can be seen that the mean values for many time slices are many standard deviations from zero for unbinned results.

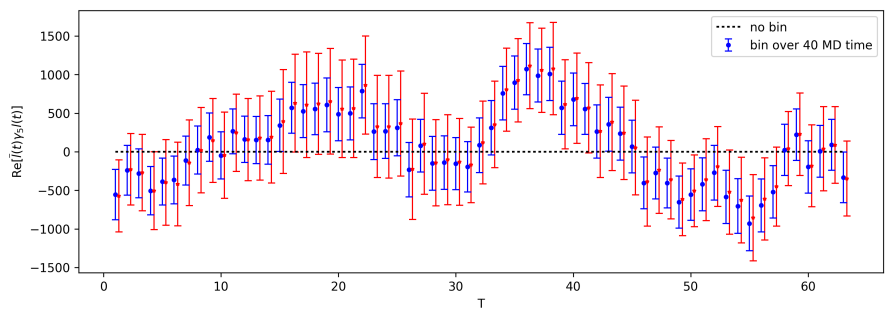

Figure 2: Closed quark loops for light quark

The reason that the mean values don't fall to zero is most likely that the autocorrelations are long. To illustrate this, we give three pieces of evidence. The first evidence comes from binned results. If one bins the results for closed quark loops over $40 \mathrm{MD}$ time units, it is found that error bars grow substantially and most mean values fall to zero (Figure 2). Also, the error bars stay the same if one bins the results over 80 MD time units, which means that the erro bars are stable for $40 \mathrm{MD}$ time units. Additional evidence for autocorrelations is that if one use only half of the trajectories (Figure 3), one finds that the mean values are very far away from zero, which shows that half of the trajectories doesn't provide enough statistics and one can deduce that the autocorrelations are long. Lastly, one can look at the evolution of one single time slice in molecular dynamic time. If one bins the results over $40 \mathrm{MD}$ time, one finds that the values could be above zero for a very long MD time. In Figure 3, the results for $\mathrm{T}=35$ is shown and one sees that it is constantly above zero for MD time 700 to 1200 . 

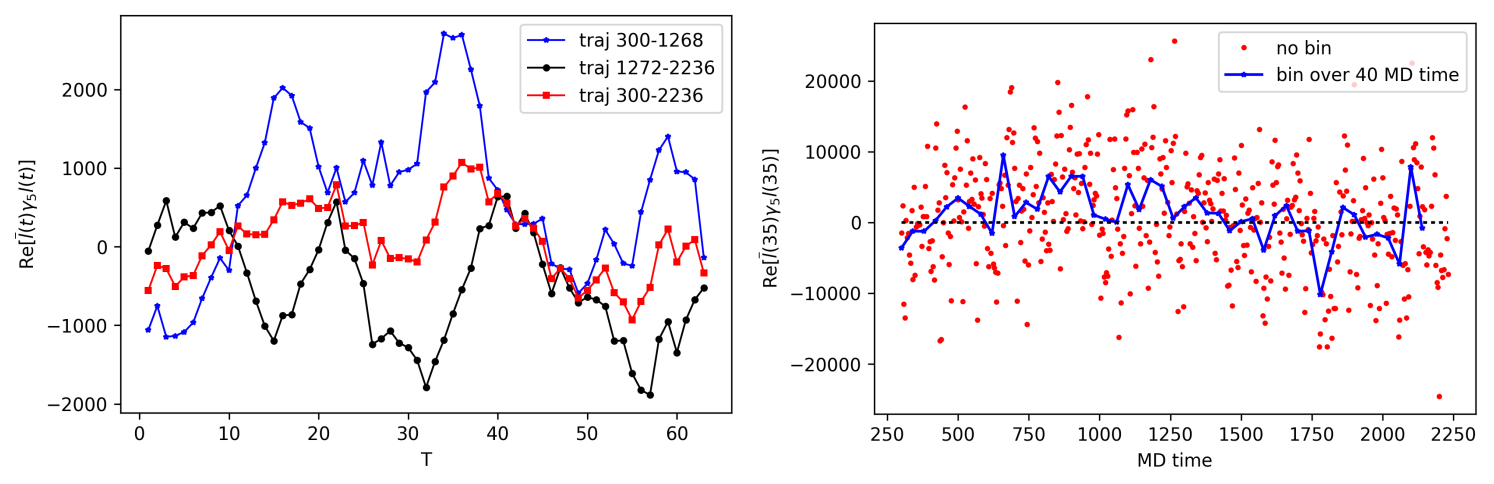

Figure 3: Left: Closed quark loops with half of the trajectories; right: Evolution of closed quark loops for one time slice

Hence, one sees that there is tension between the results of closed quark loops which probe local topological fluctuations on the configurations and the results for global topological charge measured after substantial Wilson flow. The global topological charge tells one that the gauge fields are sampled well but the closed quark loops tell one that there are long autocorrelations. Binning to 40 or $80 \mathrm{MD}$ time unites gives a better estimate of errors, but $\mathrm{O}(500) \mathrm{MD}$ time unit autocorrelations still appear.

\section{Eigenvectors}

To further investigate topological features of the configurations (without Wilson flow), we are measuring the zero-mode eigenvectors for our gauge fields. (This part of the work was not done before the lattice conference.)

One needs well-defined zero modes of the Dirac operator. We use the Hermitian operator $\gamma_{5} R_{5} D$ where $R_{5}$ is the reflection in fifth dimension and $D$ is the Shamir Dirac operator. This operator is useful because it is Hermitian and anticommutes approximately with $\Gamma_{5}$, where $\Gamma_{5}$ is the chirality operator:

$$
\left(\Gamma_{5}\right)_{s, s^{\prime}}=\delta_{s, s^{\prime}} \operatorname{sign}\left(\frac{L_{s}-1}{2}-s\right)
$$

With these properties, the chirality of the eigenvectors $\left\langle v\left|\Gamma_{5}\right| v\right\rangle$ will be close to 1 or -1 for zeromodes and 0 for non-zero modes. Thus, this provides a way to investigate individual zero-modes.

We calculated 11 eigenvectors for one configuration, configuration 976, since this configuration has asymptotic global topological charge one. In Figure 4, we plot the chirality matrix $\left\langle v_{i}\left|\Gamma_{5}\right| v_{j}\right\rangle$ and one finds that the chirality for all 11 eigenvectors are close to -1 or 1 , which means that they are zero-modes for the operator. What is interesting is that there are 6 positive and 5 negative zero-modes, or 5 positive-negative pairs. This shows that although the asymptotic global topological charge is 1 , the topological property of the gauge field is nontrivial with many topological fluctuations.

Could these zero-modes simply be artifacts concentrated on one site of the lattice? To investigate this, we plot the norm square of the eigenvectors on each time slice. They are shown in Figure 
5 and reveal that the zero-modes have radius of many lattice spacing in the $\mathrm{T}$ direction. Thus, they are physical objects from this point of view.

We are enlarging these preliminary result through measurements on more configurations. Nevertheless, these results show that the gauge field has non-trivial topological properties. It is very likely that it is not enough to look at the asymptotic global topological charge. One possible reason that the closed quark loops have long autocorrelation time is that although the net asymptotic global topological charge is changing fast, the local fields are not changing fast enough. Hence, there are long autocorrelations for each time slice.

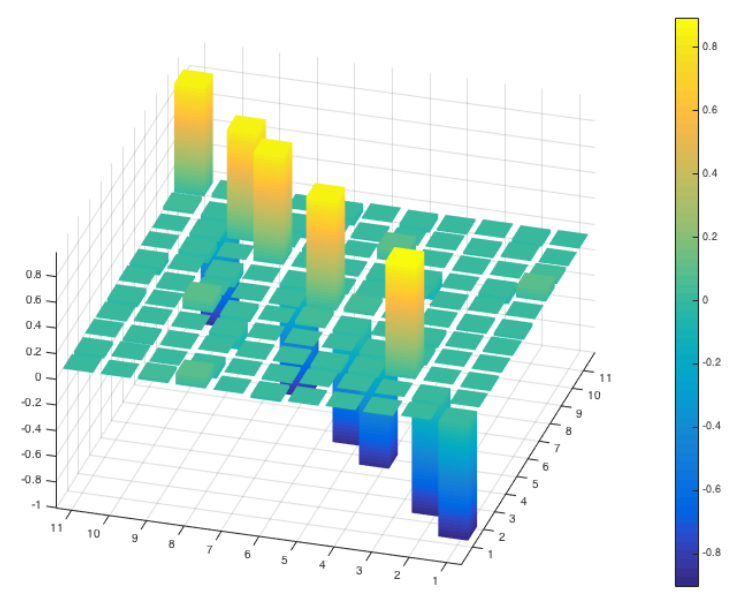

Figure 4: Chirality matrix for the first eleven eigenvectors

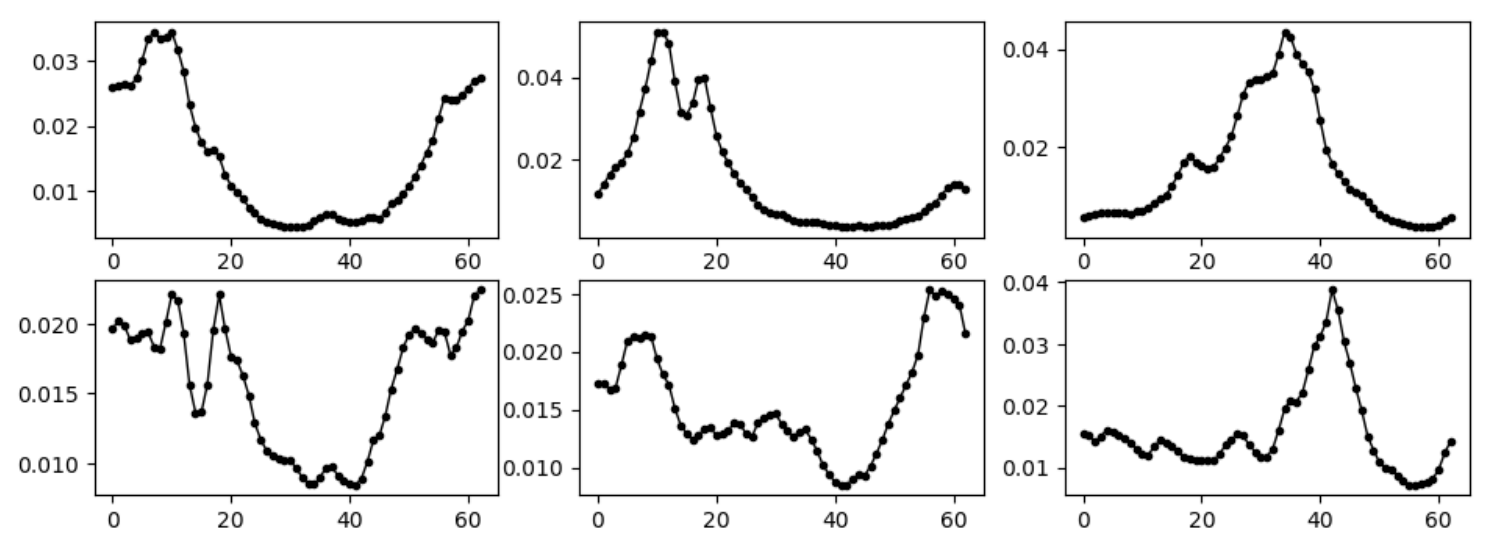

Figure 5: Norm square of the first 6 eigenvectors on each time slice

\section{5. $\eta$ and $\eta^{\prime}$ mass}

Now we give our results for $\eta$ and $\eta^{\prime}$ mass with the understanding that we likely do not have 
enough data to quote reliable errors. With approximate $\mathrm{SU}(3)$ favor symmetries:

$$
\begin{aligned}
& |\eta\rangle=\frac{1}{\sqrt{6}}\left|\bar{u} \gamma_{5} u+\bar{d} \gamma_{5} d-2 \bar{s} \gamma_{5} s\right\rangle \\
& \left|\eta^{\prime}\right\rangle=\frac{1}{\sqrt{3}}\left|\bar{u} \gamma_{5} u+\bar{d} \gamma_{5} d+2 \bar{s} \gamma_{5} s\right\rangle
\end{aligned}
$$

where $\eta$ is the octet and $\eta^{\prime}$ is the singlet. If one calculates the correlators for $\eta$ and $\eta^{\prime}$, there will be many similar terms. With $|l\rangle=\frac{1}{\sqrt{2}}\left|\bar{u} \gamma_{5} u+\bar{d} \gamma_{5} d\right\rangle$ and $|s\rangle=\left|\bar{s} \gamma_{5} s\right\rangle$, one can calculate the correlators for the light and strange state. Furthermore, the correlators consist of disconnected and connected parts [3]:

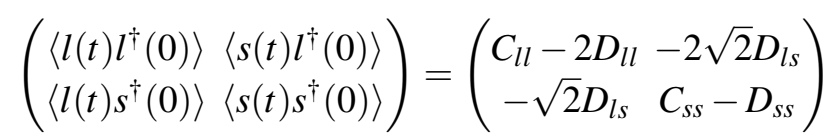

The disconnected parts are calculated directly from the closed quark loops and that's how topology enters into the mass of $\eta$ and $\eta^{\prime}$. To get the mass of $\eta$ and $\eta^{\prime}$, one can do the rotation [3]:

$$
\left(\begin{array}{l}
|\eta\rangle \\
\left|\eta^{\prime}\right\rangle
\end{array}\right)=\left(\begin{array}{cc}
\cos \theta & -\sin \theta \\
\sin \theta & \cos \theta
\end{array}\right)\left(\begin{array}{l}
|l\rangle \\
|s\rangle
\end{array}\right)
$$

With all this information, one can do simultaneous fits to get the mass of $\eta$ and $\eta^{\prime}$ [3]. The correlators are shown in Figure 6 and the fitting results are shown in Figure 7. From the correlators, one finds that the connected correlators have very small error bars but the disconnected correlators have much larger error bars, which means that the most uncertainties come from the disconnected parts.

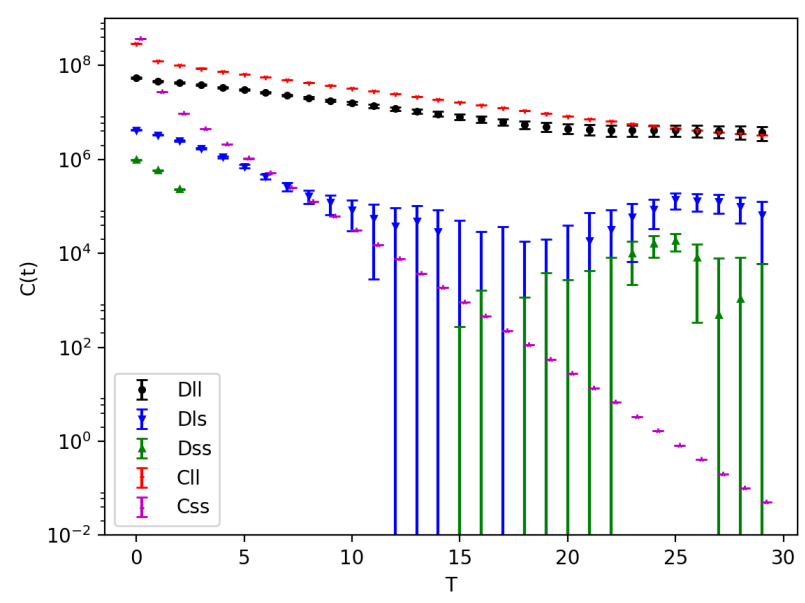

Figure 6: Disconnected and connected correlators without binning

For the fitting results, we did the fitting with different binning size: no binning, binning over $40 \mathrm{MD}$ time units and binning over $80 \mathrm{MD}$ time units. We also performed the fits with different fit ranges. From the figure, one can see that our results agree with experimental results within two standard deviations [5]. Also, increasing the binning size increases the error bars for the mass of 

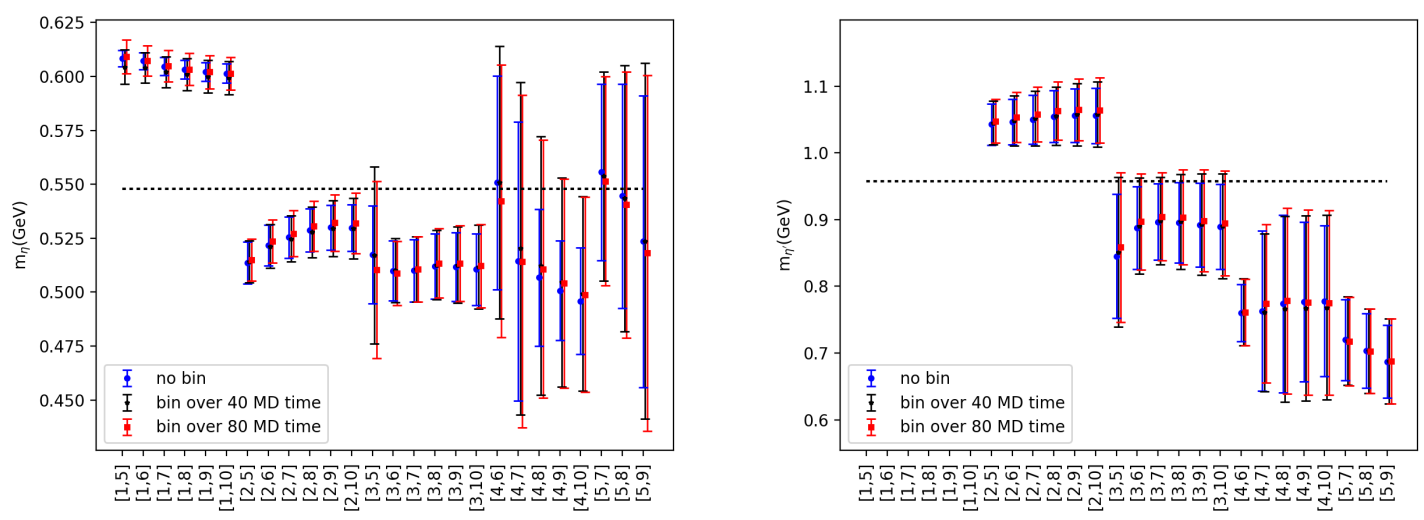

Figure 7: Results for $\eta$ and $\eta^{\prime}$ mass. The horizontal axis shows the fit range.

$\eta$. This suggests that the fitting results are not stable and more data is needed. In fact, if one recalls that the results of closed quark loops are statistically not zero, one would not expect the measurements of $\eta$ and $\eta^{\prime}$ mass to be perfect.

\section{Conclusion and Outlook}

We investigated the topological properties of the lattices from several perspectives. The asymptotic global topological charge of the lattices measured after Wilson flow is changing fast with MD time. The closed quark loops show that there are long autocorrelations for each time slice. The preliminary results from eigenvectors show that there are many topological fluctuations in the gauge field. We also measured the mass of the $\eta$ and $\eta^{\prime}$. With the data we have, the results are one or two sigma away from the experimental values. In conclusion, it is not enough to look at the asymptotic global topological charge if one wants to understand the structure of the gauge fields or fermion fields. Local properties are important for the calculation.

We are continuing to measure eigenvectors on more configurations, which will give us more statistics on the structure of the fields. We seek to see if long autocorrelations are visible in the zero-mode eigenvectors. We also plan to produce more configurations to improve the measurements of the mass of the $\eta$ and $\eta^{\prime}$.

\section{References}

[1] De Forcrand, Philippe, Margarita Garcia Perez, and Ion-Olimpiu Stamatescu. Topology of the SU (2) vacuum: a lattice study using improved cooling, Nucl.Phys, B499 409-449 (1997).

[2] G. 't Hooft, Symmetry Breaking through Bell-Jackiw Anomalies, Phys. Rev. Lett. 37, 8 (1976).

[3] N. Christ, C. Dawson, T. Izubuchi, C. Jung, Q. Liu, R. Mawhinney, C. Sachrajda, A. Soni, and R. Zhou, The $\eta$ and $\eta^{\prime}$ Mesons from Lattice QCD, Phys. Rev. Lett. 105, 241601 (2010).

[4] P. A. Boyle et al., Low Energy Constants of SU(2) Partially Quenched Chiral Perturbation Theory from $N f=2+1$ Domain Wall QCD, Phys. Rev. D93, 054502 (2016).

[5] C. Patrignani et al., Review of Particle Physics, Chin. Phys. C40, 100001 (2016). 